\title{
Sequence Heterogeneity of the ORF3 Gene of Porcine Epidemic Diarrhea Viruses Field Samples in Fujian, China, 2010-2012
}

Xi Chen ${ }^{1}$, Lili Zeng ${ }^{2}$, Jinxian Yang ${ }^{1}$, Fusong Yu ${ }^{1}$, Junqing Ge $^{1}$, Qing Guo ${ }^{2}$, Xindang Gao ${ }^{1}$ and Tieying Song ${ }^{1, *}$

1 Biotechnology Institute, Fujian Academy of Agricultural Sciences, Fuzhou 350003, China; E-Mails: kobeid@163.com (X.C.);nks139@139.com (J.Y.); yufusong58@163.com (F.Y.); junqingg@163.com (J.G.); xindanggao@163.com(X.G.)

2 Fujian Hualong Group Feed CO., LTD, Fuzhou 350003, China;

E-Mails: zenglili_2013@163.com (L.Z.); guoqinghl@126.com (Q.G.)

* Author to whom correspondence should be addressed; E-Mail: tieyingsong@163.com;

Tel.: +86-591-8781-7514; Fax: +86-591-8784-5446.

Received: 7 August 2013; in revised form: 13 September 2013 / Accepted: 19 September 2013 / Published: 30 September 2013

\begin{abstract}
Twenty-seven field samples that showed positive in PEDV detection were collected from different farms of Fujian province from 2010 to 2012. Their heterogeneity was investigated by analysis of the ORF3 gene because of its potential function as a representation of virulence. According to the results, six Fujian strains in Group 1 showed a different genotype with unique point mutations, which might be used in differentiation between PEDV groups and brought potential antigenic variation. P55 and five reference strains in Group 2 had a long length deletion, showing another genotype and might be involved in the variation of virulence. Phylogenetic analysis revealed that the collected Fujian strains were very distant from the vaccine development strain CV777, which might be the reason why the vaccine was inefficient to control the disease. The results can help to reconsider the strategy of PEDV vaccine management and prevent outbreaks of PEDV-induced diarrhea more efficiently.
\end{abstract}

Keyword: porcine epidemic diarrhea virus; PEDV; heterogeneity; phylogenetic analysis; vaccine development 
Porcine epidemic diarrhea virus (PEDV) causes severe entero-pathogenic diarrhea in piglets, especially in neonates, and the disease has a high mortality rate which can reach $80 \%$ in certain situations [1]. The disease was first recognized in England in 1971 [2], and since then, outbreaks of the disease were often reported in Europe and Asia [3-6]. Since the 1990s, a periodic vaccination strategy has been applied on pig farms nationwide to control the disease but these vaccines were not completely effective in preventing the disease, which led to a growing loss of newborn piglets in Fujian $[7,8]$.

PEDV is an enveloped, single-stranded RNA virus belonging to the family Coronaviridae [9-11]. Its genome contains six ORFs, including pplab (pol), spike (S), membrane (M), ORF3, small membrane $(\mathrm{sM})$, and nucleocapsid $(\mathrm{N})[12,13]$. The ORF3 encodes an ion channel protein and regulates virus production [14], and its loss might result in attenuation of the virus in the natural host [11]. The differentiation of ORF3 could be a marker of adaptation to cell culture and attenuation of virus [15], which could be a valuable tool to study the molecular epidemiology of PEDV [16]. The variation of field isolates of PEDV might change the genotypes and may be one of the possible reasons for the outbreaks in immunized pigs in Hebei, China [17]. Similar results were demonstrated in our primary study on field samples from 3 different swine farms in Fujian [18]. Thereby it is necessary to analyze the genetic heterogeneity of PEDV to find out which genotype prevails in Fujian. In this study, the ORF3 gene of PEDV field samples from different farms in Fujian province were cloned and sequenced for genetic diversity analysis.

Partial of intestine or stool specimens were taken individually from the acute enteritis and watery diarrhea of piglets from different big swine farms in the Fujian province between 2010 and 2012, and used for PEDV detection through PED Ag Test Kit (Bionote, Seogu-Dong, Korea). PEDV positive samples were used for sequence analysis and phylogenetic analysis. Intestinal samples were homogenized with 9 times of phosphate-buffered saline (PBS). The suspensions were then vortexed and centrifuged for $10 \mathrm{~min}$ at $1,700 \times \mathrm{g}$. The supernatants were collected and stored at $-80^{\circ} \mathrm{C}$ before utilization.

Total RNA was extracted from the supernatants of the homogenized samples with the RNAiso Plus agent (Takara, Dalian, Japan) according to the manufacturer's instructions. The forward and reverse primers [18], ORFS 5'-ACCGAGTTGAGACATACA-3' and ORFR 5'-GGAATAGAACCGTTAGACAT-3', were designed to amplify the ORF3 gene from the extracted RNA using Primescript ${ }^{\circledR}$ One Step RT-PCR Kit Ver.2 (Takara, Dalian, Japan) under the following conditions: reverse transcription at $50{ }^{\circ} \mathrm{C}$ for $30 \mathrm{~min}$, denaturation at $94{ }^{\circ} \mathrm{C}$ for $2 \mathrm{~min}, 30$ cycles of denaturation at $94{ }^{\circ} \mathrm{C}$ for $30 \mathrm{~s}$, annealing at $55^{\circ} \mathrm{C}$ for $30 \mathrm{~s}$ and extension at $72{ }^{\circ} \mathrm{C}$ for $1 \mathrm{~min}$. The RT-PCR products were analyzed by $1.5 \%$ agarose gel electrophoresis and visualized by ultraviolet illumination after ethidium bromide staining. Bands of the corresponding size of the gene were excised and the synthesized DNA was purified using QIAquick Gel Extraction Kit (QIAGEN, Dusseldorf, Germany) according to the manufacturer's instructions, then sequenced by Takara Company.

The reference strains used for the sequence analysis were described in Table 1. Alignment and phylogenetic analysis of the nucleotide sequences of the ORF3 gene were performed with ClustalW method by Mega 4.0 program [19]. The antigenic indexes of the sequence were predicted using DNAMAN program. A phylogenetic tree was constructed with nucleotide and deduced amino acid sequences using the bootstrap neighbor-joining method separately [20]. The reliability of topologies was estimated by performing bootstrap analysis with 1,000 replicates. 
Table 1. Reference porcine epidemic diarrhea virus (PEDV) strains with $99 \%$ similarities for the ORF3 genes.

\begin{tabular}{|c|c|c|}
\hline Reference strains or samples & Accession number & Source \\
\hline attenuated DR13 & JQ023162 & South Korea \\
\hline CV777 truncated ORF3 & GU372744 & Europe \\
\hline CH/GSJIII/07 & GU372743 & Heilongjiang, North China \\
\hline $\mathrm{CH} / \mathrm{BJ} / 2011$ & JQ027019 & Heilongjiang, North China \\
\hline DBI865 truncated ORF3 gene & HQ537432 & South Korea \\
\hline P55 & JQ723734 & Fujian, South China \\
\hline Zhejiang-08 & JX002703 & Zhejiang, South China \\
\hline DX & EU031893 & South Korea \\
\hline F422 & JQ723733 & Fujian, South China \\
\hline GD-A & JX112709 & Guangdong, South China \\
\hline AJ1102 & JX188454 & Hubei, North China \\
\hline $\mathrm{P} 15$ & KC120789 & Fujian, South China \\
\hline $\mathrm{P} 4$ & KC120784 & Fujian, South China \\
\hline P14 & KC120779 & Fujian, South China \\
\hline $\mathrm{P} 1$ & KC120802 & Fujian, South China \\
\hline P9 & KC120787 & Fujian, South China \\
\hline M2227 & HQ537439 & South Korea \\
\hline $\mathrm{CH} / \mathrm{S}$ & JN547228 & Heilongjiang, North China \\
\hline CH/FJND-3/2011 & JQ282909 & Heilongjiang, North China \\
\hline P37 & KC120794 & Fujian, South China \\
\hline GD-B & JX088695 & Guangdong, South China \\
\hline P718 & KC120798 & Fujian, South China \\
\hline P229 & KC120799 & Fujian, South China \\
\hline P16 & KC120790 & Fujian, South China \\
\hline $\mathrm{P} 10$ & KC120782 & Fujian, South China \\
\hline P35 & KC120793 & Fujian, South China \\
\hline P82 & KC120796 & Fujian, South China \\
\hline P17 & KC120791 & Fujian, South China \\
\hline BJ-2011-1 & JX435306 & Beijing, North China \\
\hline P83 & KC120797 & Fujian, South China \\
\hline P68 & JQ723732 & Fujian, South China \\
\hline P81 & KC120795 & Fujian, South China \\
\hline P32 & KC120792 & Fujian, South China \\
\hline P6 & KC120785 & Fujian, South China \\
\hline $\mathrm{P} 2$ & KC120783 & Fujian, South China \\
\hline P84 & KC120780 & Fujian, South China \\
\hline P19 & KC120800 & Fujian, South China \\
\hline P18 & KC120801 & Fujian, South China \\
\hline P7 & KC120786 & Fujian, South China \\
\hline P85 & KC120781 & Fujian, South China \\
\hline $\mathrm{P} 13$ & KC120788 & Fujian, South China \\
\hline CV777 & JN599150 & Europe \\
\hline LZC & EF185992 & Gansu, North China \\
\hline
\end{tabular}


In this study, 27 samples were found to show positive results in PEDV detection. They were used to amplify the ORF3 gene of PEDV, and then the gene was cloned and sequenced for alignment and phylogenetic analysis. Nucleotide sequence analysis indicated that all the samples were separated into two groups (Figure 1). The ORF3 gene of all the samples, except P55, was 675 bp in length and encoded a protein of 224 amino acids, which was similar to 10 reference strains. Six of them (F422, P1, P4, P9, P14, and P15), together with three reference strains had eight unique point mutations and formed a subgroup in Group 1. However, only one local strain, P55, was $626 \mathrm{bp}$ in length and encoded a protein of 92 amino acid, which was similar to five reference strains (CH/GSJIII/07, CV777 truncated ORF3, $\mathrm{CH} / \mathrm{BJ} / 2011$ truncated ORF3, DBI865 truncated ORF3, Zhejiang-08) in Group 2. All the Group 2 strains had a significant deletion at nt 245-294, except that the attenuated DR13 strain had a similar deletion at nt 244-294.

In terms of predicted amino acid sequence, the Subgroup 1, including six local strains, had two unique mutations, from $\mathrm{L}$ to $\mathrm{S}$ at 25 and from $\mathrm{C}$ to $\mathrm{F}$ at 107 respectively (Figure 2). It also had a unique point mutation (from $\mathrm{V}$ to $\mathrm{F}$ at 80, Figure 2), which was similar to Group 2 strains (Figure 2). P55 was found to have a long length deletion from 82 aa to the end, which existed in most of the Group 2 strains (Figure 2). No difference was found in the other 20 Fujian samples when compared to the relevant reference strains.

To analyze the phylogenetic relationships of the 27 Fujian samples and the reference strains from various parts of the world, we constructed a neighbor-joining phylogenetic tree using their ORF3 amino acid sequences (Figure 3). The results indicated that all the local stains were separated into three potential clusters, which might have different origins based on the topology. P55 was clustered into Group 2, while the others were clustered into Group 1. In Group 1, 6 Fujian samples clustered into one subgroup (Subgroup 1) and the other 20 samples were clustered into Subgroup 3.

Although the bi-combined attenuated vaccine against TGEV and PEDV infection was authorized for utilization on swine farms in Fujian province, outbreaks of PED have caused tremendous economical losses [21]. It is necessary to characterize the genetic sequence of the PEDV field samples and find out how the prevalence happened. In this study, 27 PEDV Fujian samples were identified, whose ORF3 gene sequence analysis indicated that they could be separated into two general groups. P55 with five reference strains were clustered into Group 2, which had a long length deletion in both of the nucleotide and amino acid peptide sequences. It indicated that this genotype might have prevailed in China as four strains in this group were collected from China but from different provinces (Table 1). In addition, the virulence of the virus was reduced in cell culture adaptation through the deletion mutant [15], but those five strains with the region deletion were virulent field samples, which revealed that there might be other mutations related to the virulence.

Six Fujian strains, as well as three reference ones, were characterized by eight unique point mutations in the nucleotide sequence and three amino acid changes in the peptide. These were clustered into Subgroup 1 (Figure 1). It was noteworthy that these samples were collected from four different geography regions, including South Korea (DX), Hubei in North China (AJ1102), Guangdong in South China (GD-A), and Fujian in South China (F422, P1, P4, P9, P14, and P15), respectively. Whether any recombination occurred among the field strains in these areas needs further study, but those mutations might be used in differentiation between PEDV groups or new DNA markers in the PEDV field strains. 
Figure 1. Alignment of nucleotide sequences of ORF3 genes of Fujian PEDV strains and reference strains. The asterisks represent the segments with no differences and not shown in this figure. The dashes represent deleted nucleotides. The shadows indicate the unique substitutions of chosen strains. The boxes indicate the unique insertions or deletions of chosen strains. The underlines represent the Fujian field samples in this study.

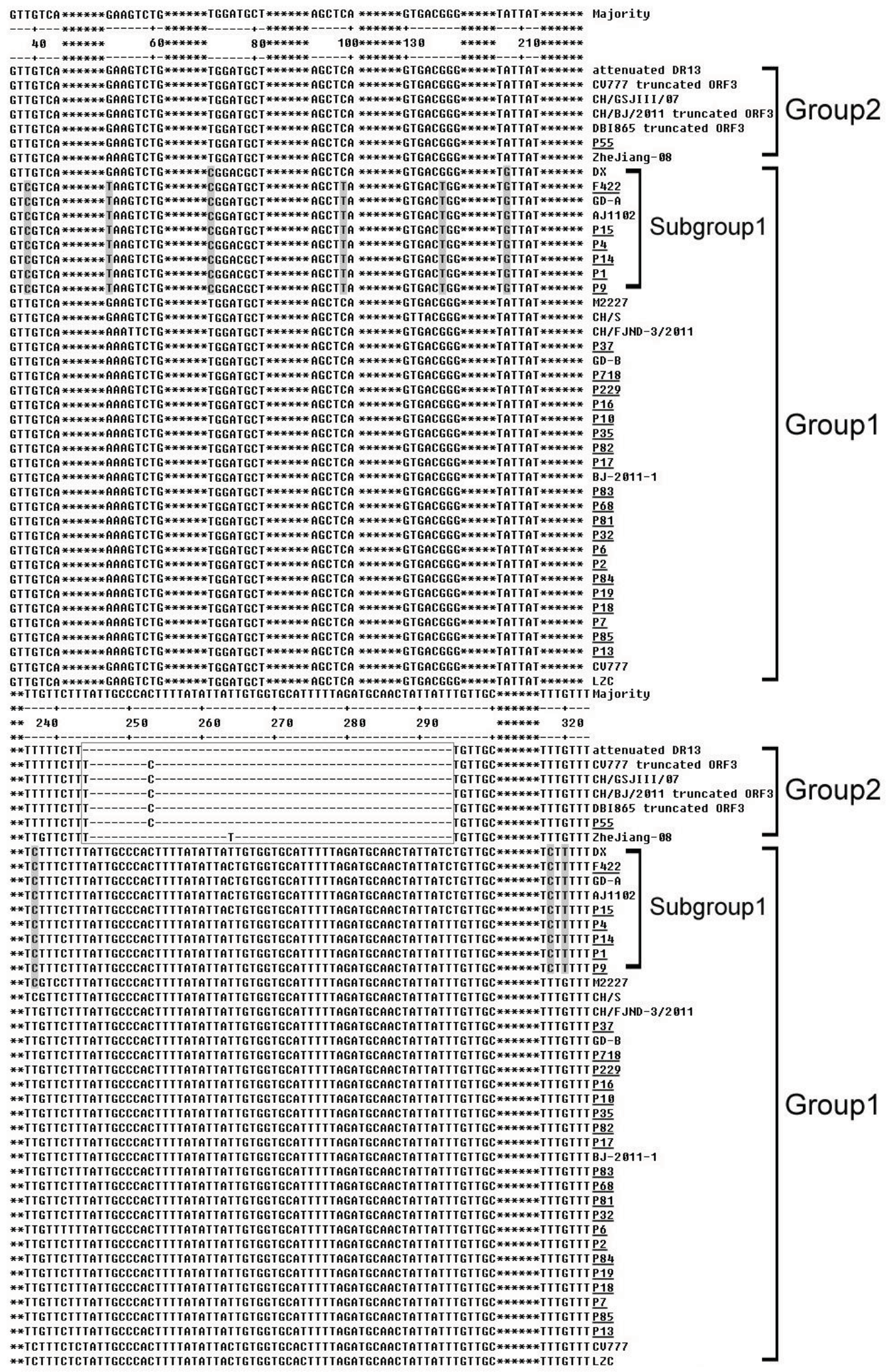


The ORF3 protein of reference strain CV777 had nine high antigenic indexes based on DNAMAN program analysis, which were located at amino acid 4-39, 41-64, 70-114, 118-124, 134-148, 158-174, 179-190, 192-207, and 211-221 plots, respectively. The strains in Subgroup 1 had amino acid changes at 25, 80 and 107 (Figure 2), located at the antigenic regions 4-39 and 70-114, respectively, which might alter their antigenicities and can be used as a label to remark this subgroup. No difference was found in the other regions.

Figure 2. Alignment of amino acid sequences of ORF3 proteins of Fujian PEDV strains and reference strains. The asterisks represent the segments with no differences and not shown in this figure. The dashes represent deleted amino acids. The shadows indicate the unique substitutions of chosen strains. The boxes indicate the unique insertions or deletions of chosen strains. The underlines represent the Fujian field samples in this study.

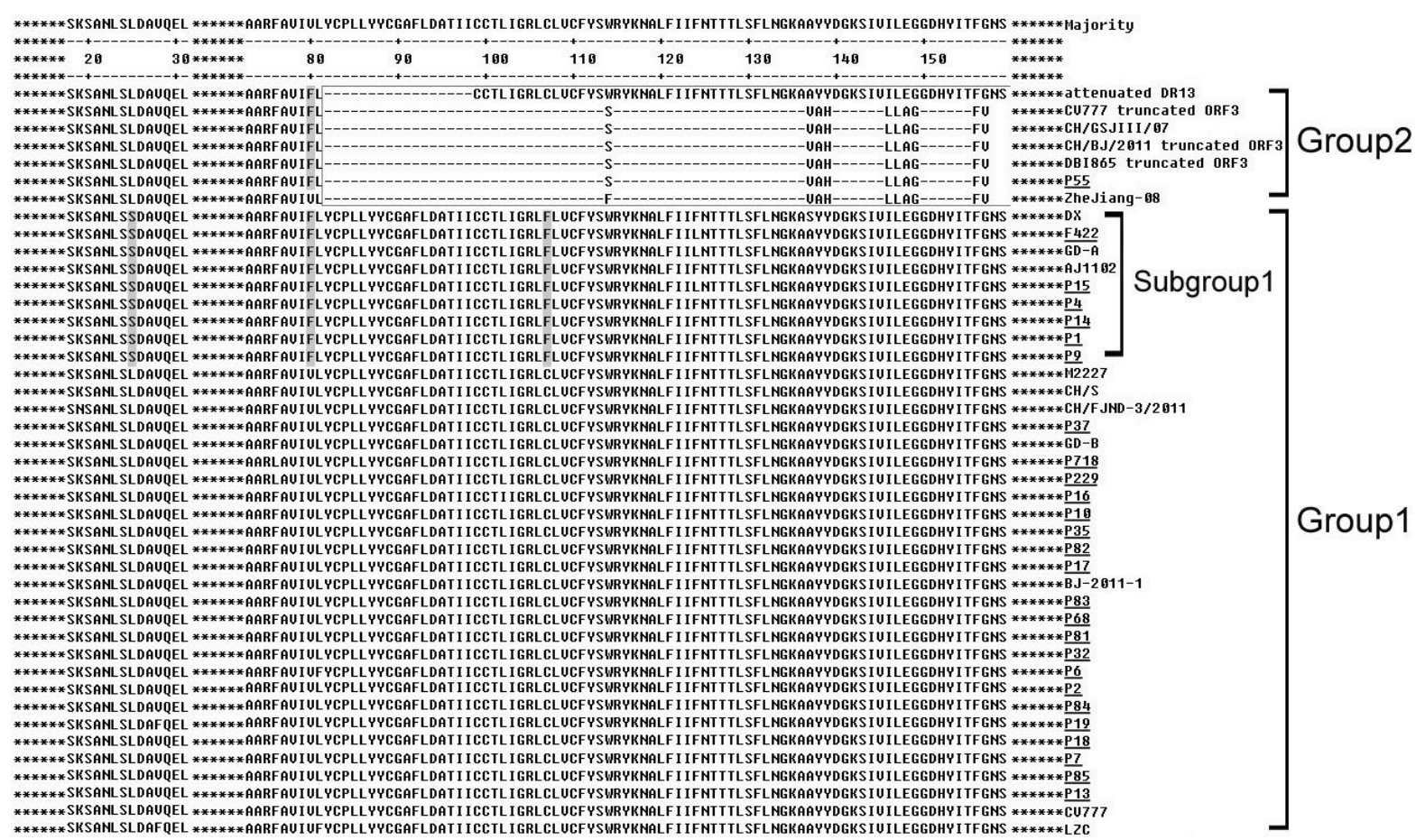

Phylogenetic analysis indicated that all the strains were divided into 2 relevant groups (Group 1 and Group 2, Figure 3), and Group 1 was formed by three subgroups. None of the Fujian strains were observed to be close to vaccine development strain, CV777 [22]. That might explain why the vaccine was not efficient enough to prevent PED prevalence. In addition, as the samples were taken from farms that covered all the district of Fujian, the phylogenetic tree demonstrated that there might be three genotypes of PEDV prevailing in Fujian.

Similar results were achieved by Li et al. [23] although they investigated a different gene (S gene) in PEDV from nine farms in 2011. In their work, three new variants were identified from field diarrhea samples, which was similar to the status of Fujian local strains (F422, P1, P4, P9, P14, and P15) in Subgroup 1 (Figures 1-3) in this study. The presence of another two field isolates, which shared high sequence identity with the attenuated strain DR13 from South Korean, was similar to P55 in the classification of Group 2 in this study (Figures 1 and 2). Both of the results demonstrated that the 
effectiveness of the CV777-based vaccine was influenced and might lead to the outbreak of severe diarrhea on China's pig farms.

Figure 3. Phylogenetic tree of the nucleotide sequences of PEDV samples based on the ORF3 gene. Sequences of reference strains were obtained from the GeneBank database. GeneBank accession numbers were shown in Table 1. Tree topology was constructed using F84 model and bootstrap re-sampling (1,000 data sets) of the multiple alignments was used to test the statistical robustness of the trees obtained by NJ (using the program Neighbor from Mega v4.0 package) G1-G4: Divided groups by phylogenetic tree. The underlines represent the Fujian field samples in this study.

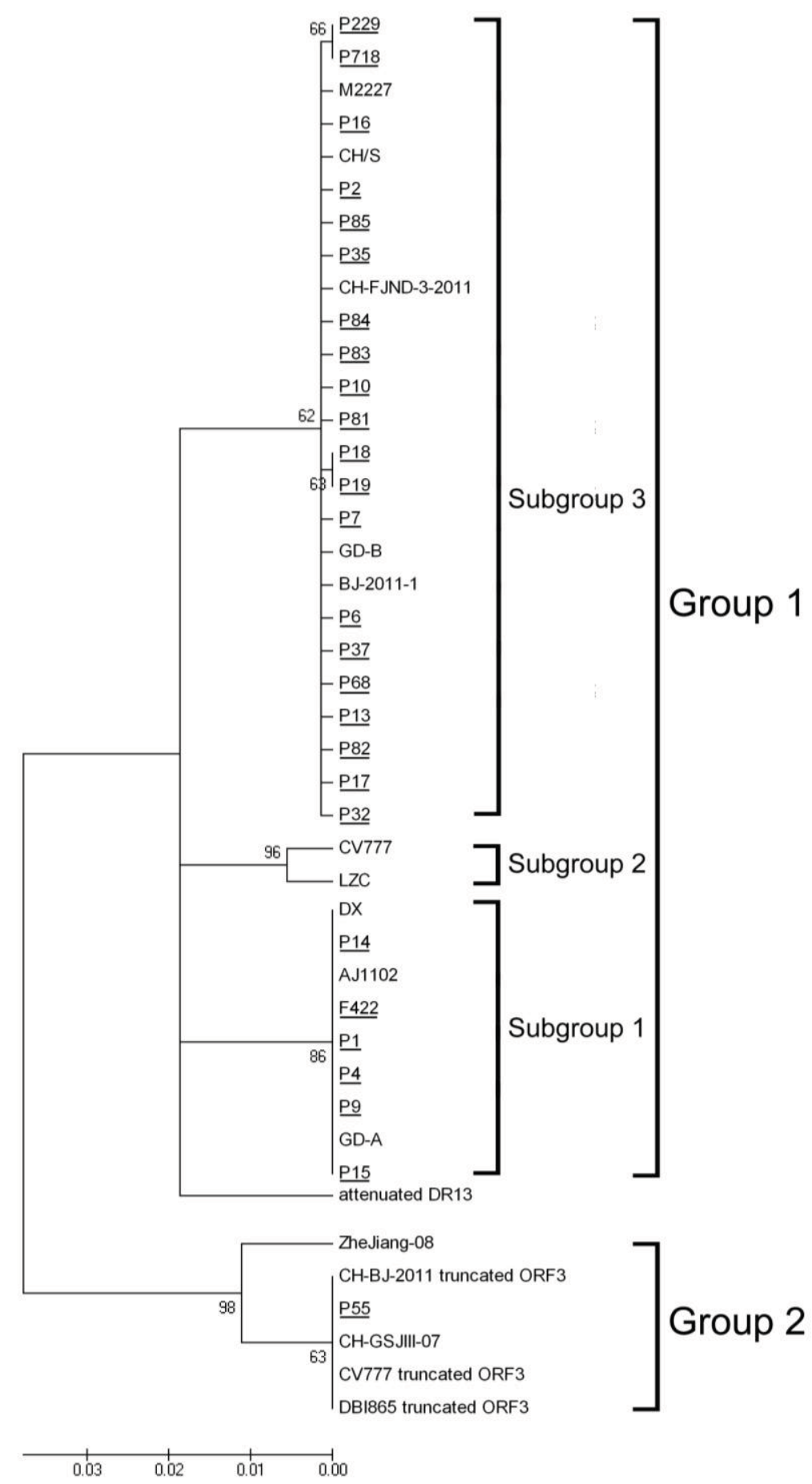


In conclusion, the PEDV field samples in Fujian province were characterized and compared by analyzing the sequence heterogeneity of ORF3 gene of PEDV. All the samples were separated into tw general groups, and the Fujian strain P55. In addition, five reference strains were clustered into Group 2, which had a long length deletion in both of the nucleotide and peptide sequences, showing a different genotype and might be involved in the variation of virulence. Six Fujian strains were clustered into Group 1, which showed another genotype with unique point mutations that might be used in differentiation between PEDV groups or new DNA markers and brought potential antigenic variation. Phylogenetic analysis revealed that the collected Fujian strains were very distant from the vaccine development strain CV777, which might be the reason why the vaccine was inefficient in controlling the disease. This study might help to choose an appropriate PEDV field strain as a vaccine candidate and efficiently prevent outbreaks of PED.

\section{Acknowledgments}

This work was supported by Project Management for Agricultural Science and Technology Achievements Transformation Fund (2010GB2C400209), Science and Technology Major Project of Fujian (2010NZ0002-3), National Spark Program (2011GA720005) and Fujian Academy of Agricultural Sciences Project (2012DQA-1).

\section{Conflicts of Interest}

The authors declare no conflict of interest.

\section{References and Notes}

1. Song, D.S.; Kang, B.K.; Lee, S.S.; Yang, J.S.; Moon, H.J.; Oh, J.S.; Ha, G.W.; Jang, Y.S.; Park, B.K. Use of an internal control in a quantitative RT-PCR assay for quantitative of porcine epidemic diarrhea virus shedding in pigs. J. Virol. Methods 2006, 133, 27-33.

2. Oldham, J. Letter to the editor. Pig Farming 1972, 10, 72-73.

3. Sato, T.; Takeyama, N.; Katsumata, A.; Tuchiya, K.; Kodama, T.; Kusanagi, K. Mutations in the spike gene of porcine epidemic diarrhea virus associated with growth adaptation in vitro and attenuation of virulence in vivo. Virus Genes 2011, 43, 72-78.

4. Pensaert, M.B.; Debouck, P.; Reynolds, D.J. An immunoelectron microscopic and immunofluorescent study on the antigenic relationship between the coronavirus-like agent, CV777, and several coronaviruses. Arch. Virol. 1981, 68, 45-52.

5. Takahashi, K.; Okada, K.; Ohshima, K. An outbreak of swine diarrhea of a new-type associated with coronavirus-like particles in Japan. Jpn. J. Vet. Sci. 1983, 45, 829-832.

6. Puranaveja, S.; Poolperm, P.; Lertwatcharasarakul, P.; Kesdaengsakonwut, S.; Boonsoongnern, A.; Urairong, K.; Kitikoon, P.; Choojai, P.; Kedkovid, R.; Teankum, K.; et al. Chinese-like strain of porcine epidemic diarrhea virus, Thailand. Emerg. Infect. Dis. 2009, 15, 1112-1115.

7. Choi, H.J.; Kim, J.H.; Lee, C.H.; Ahn, Y.J.; Song, J.H.; Baek, S.H.; Kwon, D.H. Antiviral activity of quercetin 7-rhamnoside against porcine epidemic diarrhea virus. Antivir. Res. 2009, 81, 77-81. 
8. Song, H.J.; Chae, S.W.; Yoon, K.A.; Park, J.S.; Choi, H.J. Antiviral activity of Zanthoxylum species against porcine epidemic diarrhea virus. J. Cosmet. Public Health 2010, 6, 42-44.

9. Debouck, P.; Pensaert, M.B. Experimental infection of pigs with a new porcine enteric coronavirus, CV 777. Am. J. Vet. Res. 1980, 41, 219-223.

10. Ducatelle, R.; Coussement, W.; Pensaert, M.B.; Debouck, P.; Hoorens, J. In vivo morphogenesis of a new porcine enteric coronavirus, CV777. Arch. Virol. 1981, 68, 35-44.

11. Pensaert, M.B.; Debouck, D.P. A new coronavirus-like particle associated with diarrhea in swine. Arch. Virol. 1978, 58, 243-247.

12. Bridgen, A.; Duarte, M.; Tobler, K.; Laude, H.; Ackermann, M. Sequence determination of the nucleocapsid protein gene of the porcine epidemic diarrhea virus confirms that this virus is a coronavirus related to human coronavirus $229 \mathrm{E}$ and porcine transmissible gastroenteritis virus. J. Gen. Virol. 1993, 74, 1795-1804.

13. Chang, S.H.; Bae, J.L.; Kang, T.J.; Kim, J.; Chung, G.H.; Lim, C.W.; Laude, H.; Yang, M.S.; Jang, Y.S. Identification of the epitope region capable of inducing neutralizing antibodies against the porcine epidemic diarrhea virus. Mol. Cells 2002, 14, 295-299.

14. Wang, K.; Lu, W.; Chen, J.; Xie, S.; Shi, H.; Hsu, H.; Yu, W.; Xu, K.; Bian, C.; Fischer, W.B.; et al. PEDV ORF3 encodes an ion channel protein and regulates virus production. FEBS Lett. 2012, 586, 384-391.

15. Park, S.J.; Moon, H.J.; Luo, Y.; Kim, H.K.; Kim, E.M.; Yang, J.S.; Song, D.S.; Kang, B.K.; Lee, C.S.; Park, B.K. Cloning and further sequence analysis of the ORF3 gene of wild- and attenuated-type porcine epidemic diarrhea viruses. Virus Genes 2008, 36, 95-104.

16. Chen, J.F.; Wang, C.B.; Shi, H.Y.; Qiu, H.J.; Liu, S.W.; Che, X.J.; Zhang, Z.B.; Feng, L. Molecular epidemiology of porcine epidemic diarrhea virus in China. Arch. Virol. 2010, 155, 1471-1476.

17. Fan, J.H.; Zuo, Y.Z.; Li, J.H.; Pei, L.H. Heterogeneity in membrane protein genes of porcine epidemic diarrhea viruses isolated in China. Virus Genes 2012, 45, 113-117.

18. Chen, X.; Yang, J.; Yu, F.; Ge, J.; Lin, T.; Song, T. Molecular characterization and phylogenetic analysis of porcine epidemic diarrhea virus (PEDV) samples from field cases in Fujian, China. Virus Genes 2012, 45, 499-507.

19. Tamura, K.; Dudley, J.; Nei, M.; Kumar, S. MEGA4: Molecular evolutionary genetics analysis (MEGA) software version 4.0. Mol. Biol. Evol. 2007, 24, 1596-1599.

20. Saitou, N.; Nei, M. The neighbor-joining method: A new method for reconstructing phylogenetic trees. Mol. Biol. Evol. 1987, 4, 406-425.

21. Sun, R.Q.; Cai, R.J.; Chen, Y.Q.; Liang, P.S.; Chen, D.K.; Song, C.X. Outbreak of porcine epidemic diarrhea in suckling piglets, China. Emerg. Infect. Dis. 2012, 18, 161-163.

22. Kocherhans, R.; Bridgen, A.; Ackermann, M.; Tobler, K. Completion of the porcine epidemic diarrhoea coronavirus (PEDV) genome sequence. Virus Genes 2001, 23, 137-144.

23. Li, W.; Li, H.; Liu, Y.; Pan, Y.; Deng, F.; Song, Y.; Tang, X.; He, Q. New variants of porcine epidemic diarrhea virus, China, 2011. Emerg. Infect. Dis. 2012, 18, 1350-1353.

(C) 2013 by the authors; licensee MDPI, Basel, Switzerland. This article is an open access article distributed under the terms and conditions of the Creative Commons Attribution license (http://creativecommons.org/licenses/by/3.0/). 